\title{
Generalized holographic cosmology: low-redshift observational constraint
}

\author{
Sunly Khimphun, ${ }^{a, 1}$ Bum-Hoon Lee ${ }^{b, c}$ and Gansukh Tumurtushaa ${ }^{c, d}$ \\ ${ }^{a}$ Graduate School of Science, Royal University of Phnom Penh, \\ 12150 Cambodia \\ ${ }^{b}$ Department of Physics, Sogang University, \\ Seoul, 121-742 Korea \\ ${ }^{c}$ Center for Quantum Spacetime (CQUeST), Sogang University, \\ Seoul, 121-742 Korea \\ ${ }^{d}$ Leung Center for Cosmology and Particle Astrophysics (LeCosPA), \\ National Taiwan University, Taipei 10617, Taiwan, ROC \\ E-mail: khimphun.sunly@rupp.edu.kh, bhl@sogang.ac.kr, \\ gansuh.mgl@gmail.com
}

ABSTRACT: Four-dimensional cosmological models are studied on a boundary of a fivedimensional Anti-de Sitter $\left(A d S_{5}\right)$ black hole with AdS Reissner-Nordström and scalar charged Reissner-Nordström black hole solutions, where we call the former a "Hairless" black hole and the latter a "Hairy" black hole. To obtain the Friedmann-Robertson-Walker (FRW) spacetime metric on the boundary of the $A d S_{5}$ black hole, we employ Eddington-Finkelstein (EF) coordinates to the bulk geometry. We then derive modified Friedmann equations on a boundary of the $A d S_{5}$ black hole via AdS/CFT correspondence and discuss its cosmological implications. The late-time acceleration of the universe is investigated in our models. The contributions coming from the bulk side is treated as dark energy source, and we perform MCMC analyses using observational data. Compared to the $\Lambda$ CDM model, our models contain additional free parameters; therefore, to make a fair comparison, we use the Akaike information criterion (AIC) and the Bayesian information criterion (BIC) to analyze our results. Our numerical analyses show that our models can explain the observational data as reliable as the $\Lambda \mathrm{CDM}$ model does for the current data.

KEYwords: AdS-CFT Correspondence, Holography and quark-gluon plasmas, Gaugegravity correspondence

ArXIV EPRINT: 2012.15219

\footnotetext{
${ }^{1}$ Corresponding author.
} 


\section{Contents}

1 Introduction 1

2 Five-dimensional AdS black hole and FRW boundary 2

3 Modified Friedmann equations in a $\mathbf{A d S}_{5} \quad 4$

4 Fitting models to the observational data $\quad 10$

$\begin{array}{lll}4.1 \text { Models } & 10\end{array}$

$\begin{array}{lll}4.2 \text { Data } & 11\end{array}$

4.3 MCMC analysis and model comparison 11

5 Conclusion $\quad 14$

\section{Introduction}

There are many dark energy models having been widely studied, which can be categorized as the models of $\Lambda \mathrm{CDM}$, quintessence [1-4], Chevalliear-Polarski-Linder (CPL) [5, 6], holographic principle and its observational constraints [7-16], Dvali-Gabadadze-Porrati (DGP) braneworld [17, 18], and Chaplygin gas model [19, 20, 22]. One may refer to refs. [22, 23] for various model comparisons just mentioned and [21] for a good review of many dark energy models. Many of these dark energy models are the theoretical variants of the cosmological constant model, while some are based on totally different theoretical considerations. For example, based on the slowly rolling scalar field, quintessence models produce a negative pressure for the accelerating universe. On the other hand, in the CPL model, the equation-of-state parameter is a function of time. The dark energy models based on quantum gravity theory are often regarded as the holographic models. The models in this category describe the observational data well despite its distinguished theoretical nature to $\Lambda$ CDM model. The DGP is also another interesting framework with the realization that higher-dimensional gravity affects the bulk at a large distance from which the dark energy naturally emerges. Another interesting theory is the Chaplygin gas model, which has a connection with the string theory of the braneworld scenario, whose theoretical variant so-called Generalized Chaplygin Gas (GCG) has been fitted with observational constraint [22-24]. Other classes of dark energy models which concern with $\mathrm{H}_{0}$ tensions has been extensively studied in [25-32]. Another interesting theory is based on AdS/CFT correspondence from 5-dimensional black hole [33-39]. In particular, such theory has not yet been fit with observational constraint.

In this work, we want to study cosmology from the perspective of holography; in particular, the AdS/CFT correspondence [37-39]. The dynamical evolution of the universe in four dimension can be described by the FRW metric, which arises from the boundary of $\mathrm{AdS}_{5}$ black hole. Starting from the $A d S_{5}$ black hole geometry, the FRW metric is 
realized at its four-dimensional boundary via Eddington-Finkelstein (EF) transformation. As a result, four-dimensional gravity with the dynamical FRW metric is foliated since the boundary metric can be stably set as dynamical field [41]. This holographic setting is possible mainly based on the idea of mixed-boundary condition studied in ref. [41] where such boundary condition allows the boundary metric becoming dynamical. The black hole as bulk affects the stress-energy tensor due to the AdS/CFT correspondence. Holographic renormalization is implemented in ref. [43], and for the hairy black hole case, we utilize the counterterm obtained in refs. [44, 45]. It is worth noting that such a counterterm is obtained in the Fefferman-Graham (FG) coordinate system as an intermediate steps, but the final counterterms are derived in a tensorial form. Since the nature of our study is based on boundary fields in FG coordinates which emerge from EF coordinates under the scale invariant property, we will adopt the renormalized stress-energy tensor advocated in $[44,45]$ with a slight adjustment to the scheme dependent terms. This mechanism has been studied by refs. [37-39]. In this scenario, we can treat the black hole as a higher dimensional object interacting with an ordinary gravitational theory whose effects play some roles in the cosmological evolution rather than the object where the universe resides.

The cosmological models of our interest are, therefore, extensions to the $\Lambda \mathrm{CDM}$ model. The reason is that the vacuum energy model in four-dimensional gravity theory is foliated at the boundary of one higher-dimensional spacetime. The requirement of such a fourdimensional Einstein-Hilbert action with a cosmological constant is for the purpose of the study of cosmological evolution and due to the consistent form with bare stress-energy tensor on the boundary, which admits the standard interpretation of four-dimensional constant $G_{4}$ (Newton's constant) and $\Lambda_{4}$ (cosmological constant) [38].

Moreover, we may think of this type of models as a strongly coupled field theory, but as far as an acceleration of the universe is our primary concern, we treat this as a dark energy model. As an extension, we consider a five-dimensional asymptotically anti-de Sitter(AAdS) black hole with and without a secondary scalar hair and investigate further. We derive the modified Friedmann equation for our models and compare it with $\Lambda$ CDM by using observational data, including Supernovae $[46,47]$ and $\mathrm{H}_{0}$ measurement data [48, 49]. This paper is organized as follows. In section 2, we review a procedure of obtaining the FRW metric at a boundary of the $\mathrm{AdS}_{5}$ black hole. In section 3, we derive modified Friedmann equations by employing the mixed-boundary condition and AdS/CFT correspondence, whose bulk solutions are the charged dilatonic $\mathrm{AdS}_{5}$ black hole [50]. In section 4, we present our numerical fitting results of the MCMC analyses, for which we adopt the numerical techniques developed in refs. [51-57]. We use the observational data, including Supernova (SnIa) and Hubble expansion rate data [46, 48], to provide observational bounds on model parameters associated with the late-time dynamics of the universe. Finally, section 5 is devoted to summary and conclusions of the present study.

\section{Five-dimensional AdS black hole and FRW boundary}

The idea of realizing the FRW universe at the boundary of the $\mathrm{AdS}_{5}$ black hole was first introduced in [37], where the Schwarzschild solution was considered, and subsequent works 
were done in $[38,39]$. If one can obtain a preferred boundary geometry from the AdS black hole solution, then the concept of having the mixed-boundary condition is essentially required in order to generate a dynamical FRW metric. It was shown that such a boundary condition is dynamically stable [41]. Since the new effective method used in [39] allows one to consider a class of complicated AdS black hole, in this section, we will recap such the method and consider a charged AdS dilatonic black hole solution (hairy black hole). We begin with the general metric

$$
d s^{2}=-f(r) d t^{2}+g(r) d r^{2}+\Sigma(r)^{2} d \Omega_{3}^{2} .
$$

This metric describes $\mathrm{AAdS}_{5}$ where $f(r) \sim r^{2} / L^{2}, g(r) \sim L^{2} / r^{2}$, and $\Sigma(r) \sim r / L$ for large $r$ at the asymptotic region where $L$ is the AdS radius. Introducing a new coordinates $v$ such that $d t= \pm d v / \sqrt{f(r) g(r)} \mp d r \sqrt{g(r) / f(r)}$, we obtain a metric in the EF coordinates

$$
d s^{2}=2 d v d r-f(r) d v^{2}+\Sigma(r)^{2} d \Omega_{3}^{2},
$$

which has the four-dimensional conformal boundary. We adopt a new time and radial coordinates $V$ and $R$, respectively, such that $d v=d V / a(V)$ and $R=r / a(V)$, where $a(V)$ will be the scale factor. Then the metric (2.2) becomes

$$
d s^{2}=2 d V d R-\left[\frac{f(R a)}{a^{2}}-2 R \frac{\dot{a}}{a}\right] d V^{2}+\Sigma(R a)^{2} d \Omega_{3}^{2},
$$

where the dot represents the derivative with respect to $V$. In this holographic approach to cosmology, we need to put the boundary hypersurface at a finite distance $R$ with an appropriate counterterm. As can be seen from (2.3), when large $R$ is fixed, the boundary metric reduces to FRW metric as desired. The EF coordinates associating with new time and radial coordinates is not well-understood in holographic renormalization context. For this reason, we need to find the relation between the EF and FG coordinates which is given by

$$
d s^{2}=\frac{L^{2}}{z^{2}}\left[d z^{2}+g_{\mu \nu} d x^{\mu} d x^{\nu}\right]
$$

where

$$
g_{\mu \nu}(z, x)=g_{\mu \nu}^{(0)}(x)+z^{2} g_{\mu \nu}^{(2)}(x)+z^{4}\left(g_{\mu \nu}^{(4)}(x)+h_{\mu \nu}^{(4)}(x) \log z\right)+\cdots,
$$

is defined as an appropriate form of ansatz for Fefferman-Graham asymptotic expansion [43]. Comparing the metric (2.2) with (2.4), we obtain the following two relations

$$
\begin{aligned}
2 \partial_{z} R \partial_{z} V-\alpha\left(\partial_{z} V\right)^{2} & =\frac{L^{2}}{z^{2}}, \\
\partial_{z} V \partial_{\tau} R+\partial_{\tau} V \partial_{z} R-\alpha \partial_{z} V \partial_{\tau} V & =0,
\end{aligned}
$$

where $\alpha=f(R a) / a^{2}-2 R \dot{a} / a$. The boundary metric can be obtained in the same way as

$$
g_{\tau \tau}=-\frac{\left(\partial_{\tau} V\right)^{2}}{\left(\partial_{z} V\right)^{2}}, \quad g_{i j} d x^{i} d x^{j}=\frac{z^{2}}{L^{2}} \Sigma^{2}(R a) d \Omega_{3}^{2} .
$$

The power series expansion of $V(\tau, z)$ and $R(\tau, z)$ will be obtained using (2.6) and the metric $g_{\mu \nu}$ written in terms of $z$ and $\tau$ will be determined by (2.5). 
The equation governing the cosmological evolution can be derived by using the Friedmann equation. In our study, we will obtain the modified Friedmann equations due to the contribution from higher dimension via AdS/CFT correspondence. In other words, the modified terms come from the regulated stress-energy tensor of the dual conformal field theory residing on the four-dimensional boundary hypersurface. Adopting the mixed-boundary condition, we can write our action as the following

$$
\begin{aligned}
S= & \frac{1}{16 \pi G_{5}}\left(\int_{\mathcal{M}} d^{5} x \sqrt{-\operatorname{det} g_{5}} \mathcal{L}_{5 \mathrm{D}}^{\text {gravity }}-\int_{\partial \mathcal{M}} d^{4} x \sqrt{\gamma} 2 K\right) \\
& +\frac{1}{16 \pi G_{4}} \int_{\partial \mathcal{M}} d^{4} x \sqrt{-\operatorname{det} g^{(0)}}\left(R-2 \Lambda_{4}\right)+\int_{\partial \mathcal{M}} d^{4} x \sqrt{-\operatorname{det} g^{(0)}} \mathcal{L}_{4 \mathrm{D}}^{\text {matter }}
\end{aligned}
$$

where $g_{5}$ is a five-dimensional metric, and the second integral is the Gibbons-Hawking boundary term needed to get an action that only depends on first derivatives of the metric [40]. We define $\mathcal{L}_{5 \mathrm{D}}^{\text {gravity }}$ as the Lagrangian representing the five dimensional Einstein gravity with negative cosmological constant. Notice that $g^{(0)}$ is the leading order in metric of a four dimensional boundary hypersurface corresponding to FG coordinate introduced in (2.5). Mixed-boundary condition implies that the total stress-energy tensor, which include $T_{\mu \nu}^{\mathrm{CFT}}, T_{\mu \nu}^{4 \mathrm{D}}$, and $T_{\mu \nu}^{\text {matter }}$ is zero so that the variational principle still holds [38, 41]. Thus, the five dimensional dual field theory will contributes to the stress-energy tensor and modified the equation of motion. Finally, we define $\mathcal{L}_{4 \mathrm{D}}^{\text {matter }}$ as the Lagrangian from ordinary matter. Adding appropriate counterterms to the regulated diverging action of five dimensional gravity yields renormalized stress-energy tensor defined as $\left\langle T_{\mu \nu}^{\mathrm{CFT}}\right\rangle$ so that (2.8) yields

$$
R_{\mu \nu}-\frac{1}{2} g_{(0) \mu \nu} R+\Lambda_{4} g_{(0) \mu \nu}=8 \pi G_{4}\left(\left\langle T_{\mu \nu}^{\mathrm{CFT}}\right\rangle+T_{\mu \nu}^{\text {matter }}\right)
$$

The Ricci tensor and scalar are calculated from the zeroth order boundary metric $g_{(0) \mu \nu}$. The stress-energy tensor $\left\langle T_{\mu \nu}^{\mathrm{CFT}}\right\rangle$ and $T_{\mu \nu}^{\mathrm{matter}}$ are obtained from the $\mathrm{AdS}_{5} / \mathrm{CFT}_{4}$ correspondence and from four-dimensional gravity theory with cosmological constant, respectively. The stress-energy tensor from the dual field has conformal anomaly since the boundary has an even dimension. This conformal anomaly will be remedied by the holographic renormalization.

\section{$3 \quad$ Modified Friedmann equations in a $\mathrm{AdS}_{5}$}

In this section, we begin by briefly introducing the five-dimensional scalar charged AdS black hole solution $[50,58,59]$ with the following Lagrangian in (2.8),

$$
\mathcal{L}_{5 \mathrm{D}}^{\text {gravity }}=R-W(\phi) F^{2}-\frac{1}{2}(\partial \phi)^{2}-V(\phi),
$$

with a potential $V(\phi)$ and a coupling $W(\phi)$ of the form

$$
V(\phi)=-\frac{1}{L^{2}}\left(8 e^{\phi / \sqrt{6}}+4 e^{-2 \phi / \sqrt{6}}\right), \quad W(\phi)=\frac{1}{4} e^{2 \phi / \sqrt{6}} .
$$


Scalar field which non-minimally coupled to guage field is considered here because minimal coupling scalar field will result in the trivial solution. As a result, it is out of our interest. A scalar charged Reissner-Nordström black hole solution of the given action is

$$
d s^{2}=e^{2 C}\left(-h d t^{2}+d \vec{x}^{2}\right)+\frac{e^{2 D}}{h} d r^{2}
$$

where

$$
\begin{aligned}
& C=\log \left(\frac{r}{L}\right)+\frac{1}{3} \log \left(1+\frac{Q^{2}}{r^{2}}\right), \quad D=-\log \left(\frac{r}{L}\right)-\frac{2}{3} \log \left(1+\frac{Q^{2}}{r^{2}}\right), \\
& h=1-\frac{M L^{2}}{\left(Q^{2}+r^{2}\right)^{2}}, \quad \phi=\frac{2}{\sqrt{6}} \log \left(1+\frac{Q^{2}}{r^{2}}\right), \quad A=\left(-\frac{Q \sqrt{2 M}}{Q^{2}+r^{2}}+\frac{Q \sqrt{2 M}}{Q^{2}+r_{h}^{2}}\right) d t .
\end{aligned}
$$

Here $Q$ is the charge and $M$ is the mass of the black hole. The horizon $r_{h}$ is defined such that $h\left(r_{h}\right)=0$. By introducing a new coordinate defined as follow

$$
\begin{aligned}
d t= & {\left[\frac{\dot{a} L^{2}}{a^{2} R\left(1-\frac{M L^{2}}{\left(Q^{2}+a^{2} R^{2}\right)^{2}}\right)\left(1+\frac{Q^{2}}{a^{2} R^{2}}\right)}-\frac{1}{a}\left(1+\frac{Q^{2}}{a^{2} R^{2}}\right)^{\frac{1}{3}}\right] d V } \\
& +\left[\frac{a L^{2}}{a^{2} R^{2}\left(1-\frac{M L^{2}}{\left(Q^{2}+a^{2} R^{2}\right)^{2}}\right)\left(1+\frac{Q^{2}}{a^{2} R^{2}}\right)}\right] d R .
\end{aligned}
$$

Also, one can transform the metric into the EF coordinates of the form

$$
d s^{2}=2 d v d r-h e^{-2 D} d v^{2}+e^{2 C} d \vec{x}^{2} .
$$

Comparing with the metric expression given in (2.2), we have

$$
f(r)=h e^{-2 D} \quad \text { and } \quad \Sigma(r)=e^{C} .
$$

The next step is to transform this EF coordinates to the FG coordinates for the sake of holographic renormalization. In order to do that, we first consider a power series expansion of coordinates $R$ and $V$ near $z=0$ regime which reads

$$
V(\tau, z)=\sum_{n=0} V_{(n)} z^{n}, \quad R(\tau, z)=\sum_{n=0} R_{(n)} z^{n-1} .
$$

The followings are coefficients (up to fifth order) obtained by calculating (2.6) with the given black hole geometry order by order,

$$
\begin{gathered}
V_{(0)}=\tau, \quad V_{(1)}=-1, \quad V_{(2)}=0, \quad V_{(3)}=\frac{-6 a \ddot{a}+3 \dot{a}^{2}+4 Q^{2} / L^{4}}{36 a^{2}}, \\
V_{(4)}=\frac{3 a^{2} a^{(3)}+3 \dot{a}^{3}+2 \dot{a}\left(Q^{2} / L^{4}-3 a \ddot{a}\right)}{72 a^{3}}, \\
V_{(5)}=\frac{1}{720 a^{4}}\left(-6 a^{3} a^{(4)}+24 a^{2} \ddot{a}^{2}-20 Q^{2} a \ddot{a} / L^{4}-9 \dot{a}^{4}-18 a^{2} \dot{a} a^{(3)}\right. \\
\left.+6 a \dot{a}^{2} \ddot{a}+54 M / L^{6}+10 Q^{4} / L^{8}\right) .
\end{gathered}
$$


$V_{0}=\tau$ is chosen because $V$ becomes a time $\tau$ at the boundary. Similarly, the expansion for $R$ starts from $1 / z$ and $R_{(0)}=L^{2}$ since $z \sim L^{2} / R$ near the boundary.

$$
\begin{gathered}
R_{(0)}=L^{2}, \quad R_{(1)}=\frac{L^{2} \dot{a}}{a}, \quad R_{(2)}=\frac{-6 a \ddot{a}+9 \dot{a}^{2}-2 Q^{2} / L^{4}}{12 a^{2} / L^{2}}, \\
R_{(3)}=\frac{3 a^{2} a^{(3)}+12 \dot{a}^{3}-\dot{a}\left(15 a \ddot{a}+4 Q^{2} / L^{4}\right)}{18 a^{3} / L^{2}}, \\
R_{(4)}=\frac{1}{72 a^{4}}\left(-3 a^{3} a^{(4)}+6 a^{2} \ddot{a}^{2}+10 Q^{2} a \ddot{a} / L^{4}+39 \dot{a}^{4}+21 a^{2} \dot{a} a^{(3)}\right. \\
\left.-\dot{a}^{2}\left(63 a \ddot{a}+22 Q^{2} / L^{4}\right)+9 M / L^{6}+Q^{4} / L^{8}\right),
\end{gathered}
$$

where and hereafter we set $L=1$ for simplicity. The metrics in the FG coordinates is computed (up to fourth order) applying with a profile of $V$ and $R$ in (3.9) and (3.10) to (2.5)

$$
\begin{aligned}
g_{(0) \tau \tau} & =-1, \quad g_{(2) \tau \tau}=\frac{6 a \ddot{a}-3 \dot{a}^{2}-2 Q^{2} / L^{4}}{6 a^{2}}, \\
g_{(4) \tau \tau} & =\frac{24 Q^{2} a \ddot{a}-36 L^{4} a^{2} \ddot{a}^{2}-9 L^{4} \dot{a}^{4}+\dot{a}^{2}\left(36 L^{4} a \ddot{a}-12 Q^{2}\right)+8 Q^{4} / L^{4}+108 M / L^{2}}{144 L^{4} a^{4}}, \\
g_{(0) i j} d x^{i} d x^{j} & =a^{2} d \Omega_{3}^{2}, \quad g_{(2) i j} d x^{i} d x^{j}=\left(\frac{Q^{2}}{3 L^{4}}-\frac{1}{2} \dot{a}^{2}\right) d \Omega_{3}^{2}, \\
g_{(4) i j} d x^{i} d x^{j} & =\frac{12 Q^{2} \dot{a}^{2}+9 L^{4} \dot{a}^{4}-8 Q^{4} / L^{4}+36 M / L^{2}}{144 L^{4} a^{2}} d \Omega_{3}^{2} .
\end{aligned}
$$

The zeroth order is the FRW metric as intended.

The expansion of scalar field in terms of $(\tau, z)$ is

$$
\phi(\tau, z)=\sum_{n=0} \phi_{(n)}(\tau) z^{n} .
$$

Coefficients are obtained using the scalar field given in (3.4) and listed in the following (up to sixth order).

$$
\begin{aligned}
& \phi_{(2)}=\frac{\sqrt{6} Q^{2}}{3 L^{4}} a^{2}, \quad \phi_{(4)}=\frac{Q^{2}\left(3 \dot{a}^{2}-Q^{2} / L^{4}\right)}{3 L^{4} \sqrt{6} a^{4}}, \\
& \phi_{(6)}=\frac{Q^{2}\left(27 L^{4} \dot{a}^{4}-36 Q^{2} \dot{a}^{2}-36 M / L^{2}+8 Q^{4} / L^{4}\right)}{72 \sqrt{6} L^{8} a^{6}} .
\end{aligned}
$$

Note that the zeroth order term vanishes. This is an obvious result since any field having a dual operator should vanish at the boundary.

The one form $d t$ in the gauge field $A$ in (3.4) can be converted to the EF coordinates using (3.5). Then the gauge field becomes

$$
A=A_{V} d V+A_{R} d R,
$$

where

$$
\begin{aligned}
& A_{V}=\left(-\frac{Q \sqrt{2 M}}{Q^{2}+a^{2} R^{2}}+\frac{Q \sqrt{2 M}}{Q^{2}+r_{h}^{2}}\right)\left[\frac{\dot{a} L^{2} R}{\left(1-\frac{M L^{2}}{\left(Q^{2}+a^{2} R^{2}\right)^{2}}\right)\left(Q^{2}+a^{2} R^{2}\right)}-\frac{1}{a}\left(1+\frac{Q^{2}}{a^{2} R^{2}}\right)^{\frac{1}{3}}\right], \\
& A_{R}=\left(-\frac{Q \sqrt{2 M}}{Q^{2}+a^{2} R^{2}}+\frac{Q \sqrt{2 M}}{Q^{2}+r_{h}^{2}}\right)\left[\frac{a L^{2}}{\left(1-\frac{M L^{2}}{\left(Q^{2}+a^{2} R^{2}\right)^{2}}\right)\left(Q^{2}+a^{2} R^{2}\right)}\right]
\end{aligned}
$$


The gauge field in the FG coordinates can be obtained easily from the expression in the EF coordinates.

$$
A=A_{V}\left(\frac{\partial V}{\partial \tau} d \tau+\frac{\partial V}{\partial z} d z\right)+A_{R}\left(\frac{\partial R}{\partial \tau} d \tau+\frac{\partial R}{\partial z} d z\right) \equiv A_{\tau} d \tau+A_{z} d z
$$

The power series expansions of $V$ and $R$ with respect to $z$ gives the expansion solution for $A_{\tau}$ and $A_{z}$ of the form

$$
A_{\tau}(\tau, z)=\sum_{n=0} A_{\tau}^{(n)}(\tau) z^{n}, \quad A_{z}(\tau, z)=\sum_{n=0} A_{z}^{(n)}(\tau) z^{n} .
$$

Coefficients are given as (up to fourth order)

$$
\begin{aligned}
& A_{(0) \tau}=-\frac{Q \sqrt{2 M}}{a\left(Q^{2}+r_{h}^{2}\right)}, A_{(2) \tau}=\frac{Q \sqrt{2 M}\left(3 a \ddot{a}-6 \dot{a}^{2}+6 r_{h}^{2} / L^{4}+4 Q^{2} / L^{4}\right)}{6 a^{3}\left(Q^{2}+r_{h}^{2}\right)}, \\
& A_{(4) \tau}=\frac{Q \sqrt{2 M}\left(-12 r_{h}^{2} a \ddot{a}-10 Q^{2} a \ddot{a}+36 r_{h}^{2} \dot{a}^{2}-12 \dot{a}^{4}+32 Q^{2} \dot{a}^{2}+9 L^{4} a \dot{a}^{2} \ddot{a}-8 Q^{4} / L^{4}\right)}{24 L^{4} a^{5}\left(Q^{2}+r_{h}^{2}\right)} \\
& A_{(1) z}=\frac{Q \sqrt{2 M} \dot{a}}{a^{2}\left(Q^{2}+r_{h}^{2}\right)}, A_{(2) z}=\frac{2 Q^{3} \sqrt{2 M}}{3 L^{4} a^{3}\left(r_{h}^{2}+Q^{2}\right)}, A_{(3) z}=-\frac{Q \sqrt{2 M}\left(2 r_{h}^{2} \dot{a}+2 Q^{2} \dot{a}-L^{4} \dot{a}^{3}\right)}{2 L^{4} a^{4}\left(Q^{2}+r_{h}^{2}\right)} .
\end{aligned}
$$

Before we start to consider the renormalized stress-tensor which in Maxwell field, we want to point out that the field strength tensor $F_{(0) \mu \nu}=0$ as a result of (3.18). Terms due to gauge field are the same as the minimal coupling case as the nonminimal coupling term converges to one at the boundary. As a result, all the terms given in terms of $F_{(0) \mu \nu}$ as shown in [45] will vanish. We show such term just for consistency with the action. The appropriate boundary expansion of the gauge field is [44, 45]

$$
A_{\mu}=\tilde{A}_{(0) \mu}+\tilde{A}_{(2) \mu} z^{2}+\tilde{B}_{(2) \mu} z^{2} \log z^{2}+\cdots
$$

In order to obtain the modified Friedmann equation the renormalized stress-energy tensor $T_{\mu \nu}^{\mathrm{CFT}}$ is needed. We adopt the ready-to-use equations obtained in [44] for culoumb branch flow stress-energy tensor and the current in dual theory to be

$$
\begin{aligned}
\left\langle T_{\mu \nu}^{\mathrm{CFT}}\right\rangle= & \frac{1}{4 \pi G_{5}}\left(g_{(4) \mu \nu}+\frac{1}{8}\left[\operatorname{Tr} \tilde{g}_{(2)}^{2}-\left(\operatorname{Tr} \tilde{g}_{(2)}\right)^{2}\right] g_{(0) \mu \nu}-\frac{1}{2}\left(\tilde{g}_{(2)}^{2}\right)_{\mu \nu}+\frac{1}{4} \tilde{g}_{(2) \mu \nu} \operatorname{Tr} \tilde{g}_{(2)}\right) \\
& +\frac{1}{16 \pi G_{5}}\left(\frac{1}{3}\left(\tilde{\varphi}_{(0)}^{2}-3 \varphi_{(0)} \tilde{\varphi}_{(0)}\right) g_{(0) \mu \nu}+n \varphi_{(0)}^{2} g_{(0) \mu \nu}+\frac{1}{8} \operatorname{Tr} F_{(0)}^{2} g_{(0) \mu \nu}-\frac{1}{2} F_{(0) \mu \nu}^{2}\right),
\end{aligned}
$$

and

$$
\left\langle J^{\mu}\right\rangle=\frac{1}{8 \pi G_{5}} g_{(0)}^{\mu \nu}\left(\tilde{A}_{(2) \nu}+\tilde{B}_{(2) \nu}\right)
$$

respectively. We define $\operatorname{Tr} F_{(0)}^{2}=F_{(0)}{ }^{\sigma}{ }_{\alpha} F_{(0)}{ }^{\alpha}$ and $F_{(0) \mu \nu}^{2}=F_{(0) \mu \rho} F_{(0) \nu}{ }^{\rho}$. From (3.18), we can fix $\tilde{A}_{(2) \nu}$, while $\tilde{B}_{(2) \nu}=(1 / 4) \nabla_{\rho} F_{(0) \nu}{ }^{\rho}=0$. Notice that we introduce constant $n$ to the scheme dependent term which can be adjusted after adding the local finite counterterms 
proportional to conforaml anomaly due to matter. Also, in order to adopt (3.20), which mainly base on the boundary analysis, $\tilde{g}_{(2)}$ need to be the same as pure gravity case without scalar contribution. Thus, we do not use $g_{(2) \mu \nu}$ obtained in (3.11), but it is determined by

$$
\tilde{g}_{(2) \mu \nu}=\frac{1}{2}\left(R_{\mu \nu}\left[g_{(0) \mu \nu}\right]-\frac{1}{6} R\left[g_{(0) \mu \nu}\right] g_{(0) \mu \nu}\right) .
$$

Furthermore, from boundary expansion in Einstein's equations, one cannot determined $g_{(4) \mu \nu}$ from the leading terms, but only its trace and divergence. Even though this is enough to obtain trace and divergence of $\left\langle T_{\mu \nu}^{\mathrm{CFT}}\right\rangle$, the expression in (3.20) will not provide explicit form in each component of the tensor needed for $\tau \tau$-component of the modified Friedmann equation. However, in our case, $g_{(4)}$ with scalar contribution can be determined from (3.11). First, we need to introduce the boundary expansion of $\phi$ as

$$
\phi(\tau, z)=z^{2}\left(\tilde{\varphi}_{(0)}+z^{2} \tilde{\varphi}_{(2)}\right)+z^{2} \log z^{2}\left(\varphi_{(0)}+\varphi_{(2)} z^{2}+z^{2} \log z^{2} \psi_{(2)}\right)+\cdots
$$

From (3.13), we can fix

$$
\tilde{\varphi}_{(0)}=\phi_{(2)}=\sqrt{\frac{2}{3}} \frac{Q^{2}}{a^{2} L^{4}},
$$

while $\varphi_{(0)}$ will be fixed by using the trace relation between $g_{(4)}$ and $\tilde{g}_{(2)}$. Again, from the boundary analysis [43-45],

$$
\begin{aligned}
\varphi_{(0)} & =-\frac{1}{2} \tilde{\varphi}_{(0)}=-\frac{1}{\sqrt{6}} \frac{Q^{2}}{a^{2} L^{4}} \\
\operatorname{Tr} g_{(4)}-\frac{1}{4} \operatorname{Tr} \tilde{g}_{(2)}^{2} & =-\frac{2}{3}\left(\varphi_{(0)}^{2}+2 \tilde{\varphi}_{(0)}^{2}\right)-\frac{1}{48} \operatorname{Tr} F_{(0)}^{2} \\
& =-\frac{Q^{2}}{18 a^{4} L^{4}}\left(3 a \ddot{a}-6 \dot{a}^{2}+4 Q^{2} / L^{4}\right) .
\end{aligned}
$$

It follows from (3.25) and (3.26) that

$$
\ddot{a}=\frac{1}{3 a}\left(6 \dot{a}^{2}+14 Q^{2} / L^{4}\right) .
$$

After fixing $\varphi_{(0)}, \tilde{\varphi}_{(0)}$ and using (3.27), we can see that if $n=31 / 6$ the trace of $\left\langle T_{\mu \nu}\right\rangle$ in $(3.20)$ is

$$
\begin{aligned}
\operatorname{Tr}\left\langle T^{\mathrm{CFT}}\right\rangle & =\frac{1}{48 L^{8} \pi G_{5} a^{4}}\left(5 Q^{4}-42 L^{4} \dot{a}^{2} Q^{2}-18 L^{8} \dot{a}^{4}\right)-\frac{1}{64 \pi G_{5}} \operatorname{Tr} F_{(0)}^{2} \\
& =-2 \varphi_{(0)}\left\langle\mathcal{O}_{\phi}\right\rangle+\mathcal{A}_{g}+\mathcal{A}_{\phi}
\end{aligned}
$$

where

$$
\mathcal{A}_{g}=\frac{1}{16}\left(R_{\mu \nu} R^{\mu \nu}-\frac{1}{3} R^{2}\right), \quad \mathcal{A}_{\phi}=2 \varphi_{(0)}^{2},
$$

which are the correct trace anomaly due to gravity and matter respectively, and $\left\langle\mathcal{O}_{\phi}\right\rangle=2 \tilde{\varphi}_{(0)}$ is the scalar operator $\left\langle\mathcal{O}_{\phi}\right\rangle=2 \tilde{\varphi}_{(0)}$ obtained in [42], and the last term in (3.28), just to clarify, comes from boundary expansion in (3.26), but not a result from the traceless Maxwell 
stress tensor in (3.20). Fixing $\varphi_{(0)}, \tilde{\varphi}_{(0)}$, and $n$ allow us obtain the covariant divergence of $g_{(4)}$,

$$
\begin{aligned}
\nabla^{\nu} g_{(4) \mu \nu}= & \frac{1}{4} \nabla^{\nu}\left(-\frac{1}{8}\left[\operatorname{Tr} \tilde{g}_{(2)}^{2}-\left(\operatorname{Tr} \tilde{g}_{(2)}\right)^{2}\right] g_{(0) \mu \nu}+\frac{1}{2}\left(\tilde{g}_{(2)}^{2}\right)_{\mu \nu}-\frac{1}{4} \tilde{g}_{(2) \mu \nu} \operatorname{Tr} \tilde{g}_{(2)}\right. \\
& \left.-\frac{1}{3}\left(\tilde{\varphi}_{(0)}^{2}-\frac{33}{2} \varphi_{(0)}^{2}\right) g_{(0) \mu \nu}-\frac{1}{\sqrt{6} \tilde{\varphi}_{(0)}}\left(\nabla_{\mu} \tilde{\varphi}_{(0)} \nabla_{\nu} \tilde{\varphi}_{(0)}\right)\right) \\
- & \nabla^{\nu}\left(\frac{1}{48} \operatorname{Tr} F_{(0)}^{2} g_{(0) \mu \nu}\right)+\frac{1}{2}\left(A_{(2) \nu}+B_{(2) \nu}\right) g_{(0)}^{\nu \rho} F_{(0) \mu \rho} .
\end{aligned}
$$

Using (3.20), (3.21) and (3.30), one can check that

$$
\nabla^{\mu}\left\langle T_{\mu \nu}^{\mathrm{CFT}}\right\rangle=-\frac{Q^{4} \dot{a}}{12 \pi L^{8} a^{5} G_{5}}=-\left\langle\mathcal{O}_{\phi}\right\rangle \nabla_{\nu} \varphi_{(0)}+F_{(0) \mu \nu}\left\langle J^{\mu}\right\rangle
$$

Notice that there is no term $\left\langle J^{\mu}\right\rangle A_{(0) \mu}$ in (3.28) and $A_{(0) \nu} \nabla_{\mu}\left\langle J^{\mu}\right\rangle$ in (3.31) because $\left\langle T_{\mu \nu}^{\mathrm{CFT}}\right\rangle$ in (3.20) does not explicitly depend on the source $A_{(0) \mu}$. Using (3.18) and (3.21) we obtain

$$
\nabla^{\mu}\left\langle J_{\mu}\right\rangle=\frac{Q(a \dddot{a}-3 \dot{a} \ddot{a})}{8 \sqrt{2} L \pi a^{3} G_{5}}
$$

so the current of the dual field theory is not conserved which is due to $A_{(0) \tau}$ playing a role of dynamical chemical potential. Notice that we have used $r_{h}^{2}=L \sqrt{M}-Q^{2}$. Using (3.20) and (3.27), the explicit formula for the stress-energy tensor and dual charge is

$$
\begin{aligned}
\left\langle T_{\tau \tau}^{\mathrm{CFT}}\right\rangle & =\frac{9 L^{8} \dot{a}^{4}+12 L^{4} Q^{2} \dot{a}^{2}+36 L^{2} M+23 Q^{4}}{192 \pi a^{4} \mathrm{G} 5 L^{8}}, \\
\left\langle T_{i j}^{\mathrm{CFT}}\right\rangle & =\frac{-63 L^{8} \dot{a}^{4}-156 L^{4} Q^{2} \dot{a}^{2}+36 L^{2} M+31 Q^{4}+12 Q^{4}}{576 L^{8} \pi a^{2} G_{5}} \delta_{i j} .
\end{aligned}
$$

The energy density, pressure and charge density $\mathcal{Q}=\left\langle J^{\tau}\right\rangle$ read

$$
\begin{aligned}
\left\langle\rho^{\mathrm{CFT}}\right\rangle & =\frac{9 L^{8} \dot{a}^{4}+12 L^{4} Q^{2} \dot{a}^{2}+36 L^{2} M+23 Q^{4}}{192 \pi a^{4} \mathrm{G} 5 L^{8}}, \\
\left\langle p^{\mathrm{CFT}}\right\rangle & =\frac{-63 L^{8} \dot{a}^{4}-156 L^{4} Q^{2} \dot{a}^{2}+36 L^{2} M+31 Q^{4}+12 Q^{4}}{192 L^{8} \pi a^{2} G_{5}} . \\
\mathcal{Q} & =\frac{Q\left(3 a L^{4} \ddot{a}-6 L^{4} \dot{a}^{2}+6 L \sqrt{M}-2 Q^{2}\right)}{24 \sqrt{2} L^{5} \pi G_{5} a^{3}}
\end{aligned}
$$

The $\tau \tau$-component of the Einstein equation in (2.9) gives a modified Friedmann equation by using (3.20) and (3.27)

$$
\left(1-\frac{\beta Q^{2}}{6 a^{2} L^{4}}\right) H^{2}=\frac{\beta}{8}\left(\frac{36 L^{2} M+23 Q^{4}}{9 a^{4} L^{8}}+H^{4}\right)+\frac{8 \pi G_{4}}{3} \rho+\frac{\Lambda_{4}}{3},
$$

where $H=\dot{a} / a$ and $\beta=G_{4} / G_{5}$. Similarly, the $i j$-component of (2.9) gives

$$
\frac{7 \beta}{24} H^{4}+\left(\frac{13 \beta Q^{2}}{18 a^{2} L^{4}}-\frac{5}{3}\right) H^{2}=\beta\left(\frac{36 L^{2} M+43 Q^{4}}{216 a^{4} L^{8}}\right)+\frac{8 \pi G_{4}}{3} p-\frac{\Lambda_{4}}{3}
$$


Notice that $i j$-component can also be derived from $\tau \tau$-component and the trace of $(2.9)$. The trace is

$$
\frac{\beta}{2} H^{4}+\left(\frac{7 \beta Q^{2}}{6 a^{2} L^{4}}-3\right) H^{2}=\frac{5 \beta Q^{4}}{36 L^{8} a^{4}}+\frac{14 Q^{2}}{3 L^{4} a^{2}}+\frac{4 \pi G_{4}}{3}(3 p-\rho)-\frac{2 \Lambda_{4}}{3},
$$

which can be another check for stress-energy tensor in (3.20). In the $Q \rightarrow 0$ limit, the scalar field and the gauge field vanish and the potential becomes a cosmological constant in the five dimensional AdS. This means that the hairy black hole geometry reduces to the AdS-Schwarzschild black hole geometry. The modified Friedmann equation (3.35) should also reduce to the one derived from the five-dimensional AdS-Schwarzschild black hole. The relevant equation has been derived in [37] (note that there is a typo in the original paper) and is found to be the same with the (3.35) in the $Q \rightarrow 0$ limit. Terms proportional to $\beta$ on the right hand side of each equation are from the conformal field theory dual to the gravity. If there are no such terms, the equation falls into the standard expression, the Friedman equation in the $\Lambda$ CDM model.

One last thing worthwhile to mention here is the temperature of the universe. As the black hole in the bulk has the finite Hawking temperature $T_{H}$, this contributes to the boundary temperature. As the radial coordinates was scaled when we choose the boundary, the temperature of the universe is also scale by the scale factor $a(V)$. So the temperature of the universe has additional $T_{H} / a(V)$ term. The overall temperature of the boundary attributes to the bulk black hole and the real particles on the boundary.

\section{Fitting models to the observational data}

In this section, using the modified Friedmann equation (3.35) and numerical techniques developed in [52], we test our model in (3.1) against the observational data and present our results of MCMC analysis.

\subsection{Models}

The evolution of the universe for our model is governed by the modified Friedmann equation (3.35), which can be rewritten as

$$
\begin{aligned}
0= & \frac{H^{4}}{H_{0}^{4}}-\left[\frac{8}{\beta H_{0}^{2}}-\frac{4 Q^{2}}{3 L^{4} H_{0}^{2}}(1+z)^{2}\right] \frac{H^{2}}{H_{0}^{2}}+\left(\frac{36 L^{2} M+23 Q^{4}}{9 L^{8} H_{0}^{4}}\right)(1+z)^{4} \\
& +\frac{8}{\beta H_{0}^{2}}\left[\Omega_{r}(1+z)^{4}+\Omega_{m}(1+z)^{3}+\Omega_{\Lambda}\right],
\end{aligned}
$$

where $a(\tau)=a\left(\tau_{0}\right) /(1+z)$ is used. The solution for above equation is

$$
\frac{H^{2}}{H_{0}^{2}}=\frac{1}{2 \Omega_{\beta}}\left[1-\Omega_{Q}(1+z)^{2} \pm \sqrt{\left[1-\Omega_{Q}(1+z)^{2}\right]^{2}-4 \Omega_{\beta}\left[\tilde{\Omega}_{r}(1+z)^{4}+\Omega_{m}(1+z)^{3}+\Omega_{\Lambda}\right]}\right],
$$

where $\tilde{\Omega}_{r} \equiv \Omega_{r}+\Omega_{M}$ and the density parameters are defined as

$$
\Omega_{\beta} \equiv \frac{\beta H_{0}^{2}}{8}, \Omega_{Q} \equiv \frac{4 Q^{2}}{3 L^{4} H_{0}^{2}} \Omega_{\beta}, \Omega_{M} \equiv \frac{36 L^{2} M+23 Q^{4}}{9 L^{8} H_{0}^{4}} \Omega_{\beta}, \Omega_{m, r} \equiv \frac{8 \pi G_{4} \rho_{m, r}^{0}}{3 H_{0}^{2}}, \Omega_{\Lambda} \equiv \frac{\Lambda_{4}}{3 H_{0}^{2}},
$$


and $H_{0}$ is the current value of the Hubble parameter, $H_{0}=100 h \mathrm{~km} \mathrm{~s}^{-1} \mathrm{Mpc}^{-1}$. By taking (4.2) at $z=0$, i.e., $\left.H_{0} \equiv H(z)\right|_{z=0}$, we obtain a relation between different energy components as follows

$$
1=\frac{1}{2 \Omega_{\beta}}\left[\left(1-\Omega_{Q}\right) \pm \sqrt{\left(1-\Omega_{Q}\right)^{2}-4 \Omega_{\beta}\left(\Omega_{M}+\Omega_{r}+\Omega_{m}+\Omega_{\Lambda}\right)}\right] .
$$

We obtain from the last equation that

$$
\Omega_{\Lambda}=1-\Omega_{m}-\tilde{\Omega}_{r}-\Omega_{Q}-\Omega_{\beta}
$$

where we introduced $\tilde{\Omega}_{r}$ due to the fact that they have the same evolution hence the number of free parameter reduces by one.

\subsection{Data}

In our numerical analysis, we use two different observational data sets from the low-redshift measurements including the Supernovae Type Ia (SnIa) and the direct measurements of the Hubble expansion rate. In particular, we use the Pantheon compilation of SnIa data [46] and the cosmic chronometric data on $H(z)$ [48]. There are two ways of deriving $H(z)$; by the clustering of galaxies or quasars and by the differential age method. The first method provides direct measurements of the $H(z)$ by measuring the BAO peak in the radial direction from the clustering of galaxies or quasars [60] while the second method obtains the $H(z)$ via the redshift drift of distant objects over significant time periods, which is possible as in GR the $\mathrm{H}(\mathrm{z})$ can be expressed in terms of change in the redshift, i.e., $H(z)=-1 /(1+z) d z / d t[61]$. As a result, these methods provide 36 data points of the $H(z)$ between $0.07 \leq z \leq 2.36$. We then compute the total likelihood function $\mathcal{L}_{\text {tot }}$, which can be written as the product of likelihood functions of each data set, $\mathcal{L}_{\text {tot }}=\mathcal{L}_{\text {SnIa }} \times \mathcal{L}_{H_{0}}$. The likelihood function can be converted into the sum of the total $\chi^{2}, \chi_{\text {tot }}^{2}=\chi_{\text {SnIa }}^{2}+\chi_{H_{0}}^{2}$, where $\chi_{\text {tot }}^{2}=-2 \log \mathcal{L}_{\text {tot }}$ is used. In the following, let us explain the data sets used in the likelihood analysis.

\subsection{MCMC analysis and model comparison}

By using on the $\chi^{2}$ functions for each data set, we perform a MCMC sampling analysis for the cosmological parameters including $\Omega_{m}, \Omega_{b} h^{2}, h, \Omega_{\beta}$, and $\Omega_{Q}$. We plot one-dimensional probability distribution and two-dimensional observational contours in figure 1 and 2 for both hairless and hairy $\mathrm{BH}$ cases, respectively. The main results regarding the best-fit values are listed in table 1 in comparison to that of the $\Lambda \mathrm{CDM}$ model.

The $\Lambda$ CDM model, which is currently regarded as the best cosmological model explaining the observational data among all existing ones, can be recovered in our study when $\Omega_{\beta}=0$. Thus, our models on the four-dimensional conformal boundary of the $A d S_{5} \mathrm{BH}$ can be treated as a simple extension to the $\Lambda \mathrm{CDM}$ model hence the direct comparison between the models can be done.

In regard to comparing the statistical significance our model with the $\Lambda \mathrm{CDM}$ model, $\chi_{\min }^{2}$ cannot make a fair comparison because of the fact that a model with more parameters 


\begin{tabular}{l|l|l|l}
\hline Parameters & $\Lambda$ CDM & Hairless BH model & Hairy BH model \\
\hline$\Omega_{m}$ & $0.2848 \pm 0.0187$ & $0.2872 \pm 0.0191$ & $0.2852 \pm 0.0141$ \\
$h$ & $0.6853 \pm 0.0169$ & $0.6849 \pm 0.0181$ & $0.6855 \pm 0.0143$ \\
$\Omega_{\beta}$ & - & $(5.5431 \pm 2.6235) \times 10^{-4}$ & $(5.1275 \pm 2.6600) \times 10^{-4}$ \\
$\Omega_{Q}$ & - & - & $(2.3937 \pm 1.2845) \times 10^{-3}$ \\
\hline$\chi_{\min }^{2}$ & 1056.56 & 1056.62 & 1056.81 \\
$\Delta$ AIC & 0 & 2.06 & 4.25 \\
$\Delta$ BIC & 0 & 7.01 & 14.15 \\
\hline
\end{tabular}

Table 1. The best-fit values of cosmological parameters and their uncertainties with $68.3 \%$ C.L.

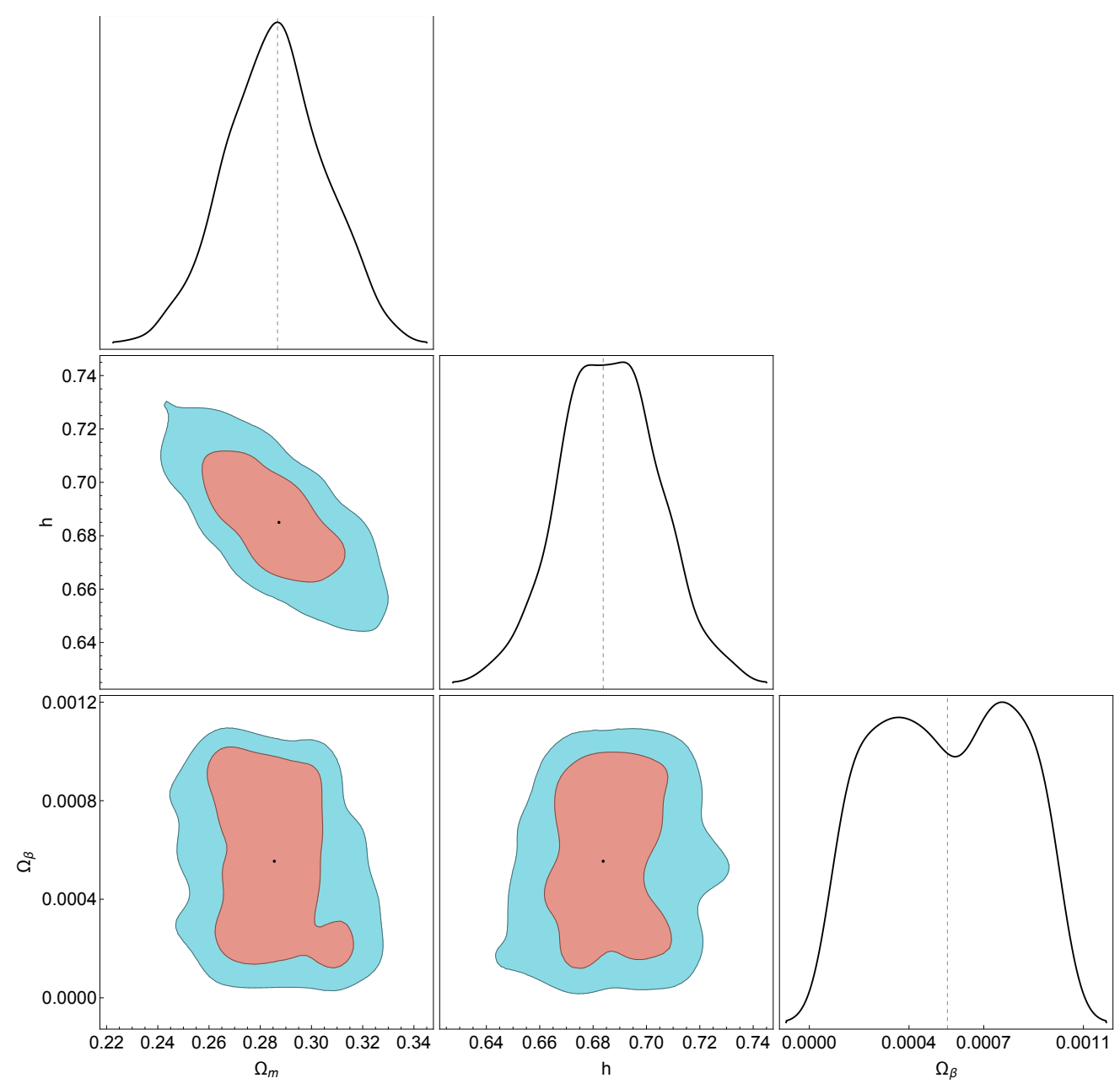

Figure 1. The $68.3 \%$ and $95.4 \%$ confidence contours between parameters for the Hairless BH case and their 1D marginalized likelihood. The vertical dashed lines and black dots indicate the mean MCMC values at $\left(\Omega_{m}, h, \Omega_{\beta}\right)=\left(0.2855,0.6837,5.5431 \times 10^{-4}\right)$. 


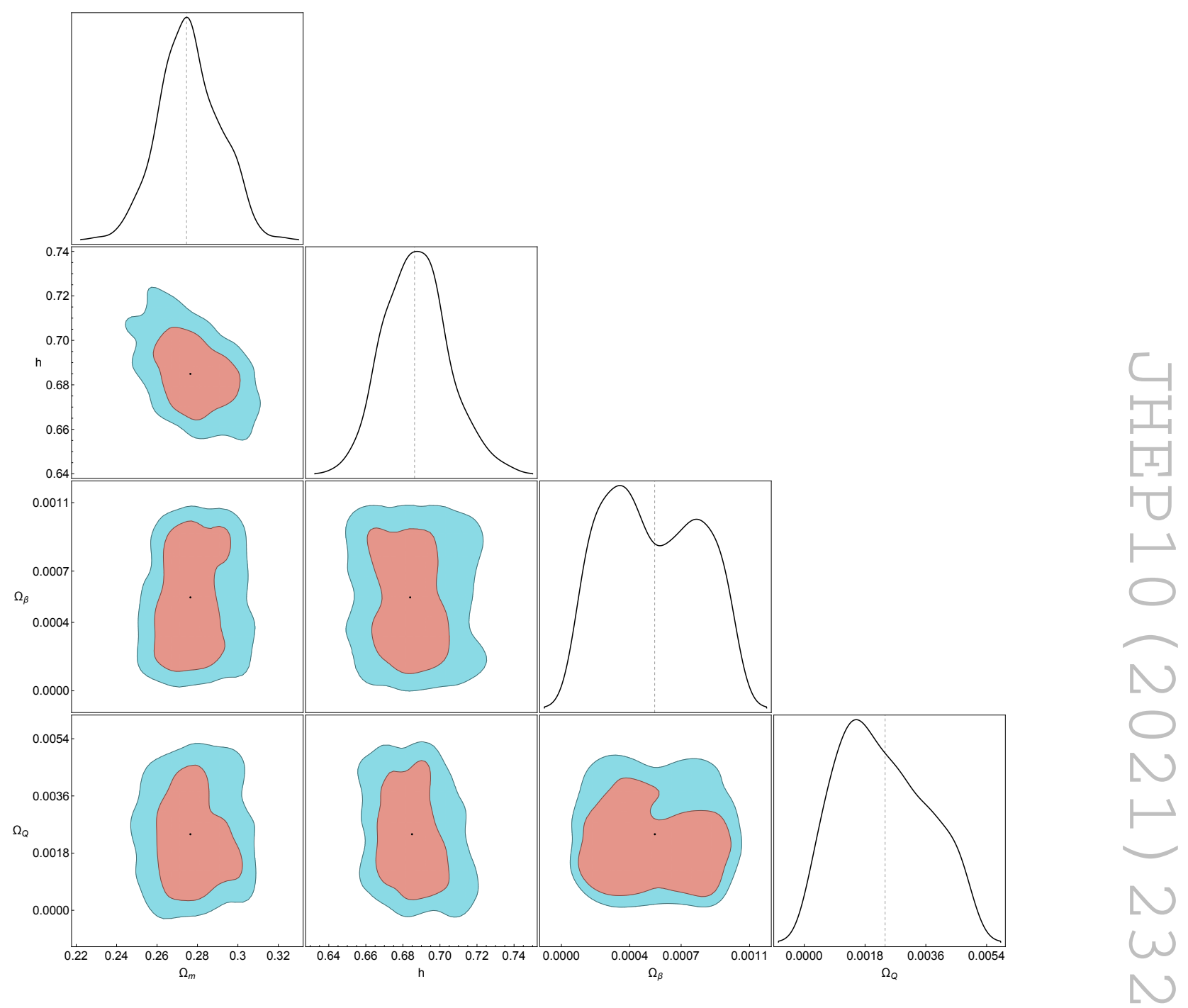

Figure 2. The $68.3 \%$ and $95.4 \%$ confidence contours between parameters for the Hairy BH case and their 1D marginalized likelihood. The vertical dashed lines and black dots indicate the mean MCMC values at $\left(\Omega_{m}, h, \Omega_{\beta}, \Omega_{Q}\right)=\left(0.2765,0.6849,5.4624 \times 10^{-4}, 2.3937 \times 10^{-3}\right)$.

has more tendency to have a lower value of $\chi_{\min }^{2}$ in general. Compared to the $\Lambda$ CDM model, our models have one additional parameter $\left(\Omega_{\beta}\right)$ for the hairless BH case and two $\left(\Omega_{\beta}\right.$ and $\left.\Omega_{Q}\right)$ more for the hairy BH case. Thus, in order to make a fair comparison, we use well known Akaike information criterion (AIC) [62] and Bayesian information criterion (BIC) [63] in our study.

The AIC and BIC estimators are defined as $\mathrm{AIC} \equiv-2 \ln \mathcal{L}_{\max }+2 k$ and $\mathrm{BIC} \equiv$ $-2 \ln \mathcal{L}_{\text {max }}+k \ln N$, where $\mathcal{L}_{\max }, k$, and $N$ indicate the maximum likelihood, the number of free parameters, and the number of data points we use in our model-to-data fitting, respectively. Assuming Gaussian errors, one can use $\chi_{\min }^{2}=-2 \ln \mathcal{L}_{\max }$. The usual interpretation of the AIC and BIC estimator is that a model with a smaller AIC value means a better model in terms of data fitting, while a smaller BIC value indicates that such a model 
is economically favorable if further data points are implemented. The $\Lambda \mathrm{CDM}$ is used as a reference model in our study. Thus, we need to use the pair difference between our model and $\Lambda \mathrm{CDM}$ model; $\Delta \mathrm{AIC}=\mathrm{AIC}_{\text {our model }}-\Delta \mathrm{AIC}_{\Lambda \mathrm{CDM}}$ and $\Delta \mathrm{BIC}=\mathrm{BIC}_{\text {our model }}-\Delta \mathrm{BIC}_{\Lambda \mathrm{CDM}}$. This can be translated as $\Delta \mathrm{AIC}=\Delta \chi_{\min }^{2}-2 \Delta k$ and $\Delta \mathrm{BIC}=\Delta \chi_{\min }^{2}-\Delta k \ln N$, respectively.

The $\Delta \mathrm{AIC}$ and $\Delta \mathrm{BIC}$ can be interpreted similarly to the $\chi_{\min }^{2}$, i.e., a relative value signifies a better fit to data. In other words, this relative difference can be interpreted with the Jeffreys' scale as follows: $0<\Delta \mathrm{AIC} \leq 2$ indicates the consistency between two models, $4<\Delta$ AIC $<7$ suggests a positive evidence against the model with higher value of $\mathrm{AIC}_{\text {model }}$, and $\triangle \mathrm{AIC}>10$ can be interpreted as an indication of essentially no support with respect to the reference model. For the BIC, the relative difference $\Delta \mathrm{BIC}=\mathrm{BIC}_{\text {model }}-\mathrm{BIC}_{\Lambda \mathrm{CDM}}$ provides the following situations: $\triangle \mathrm{BIC} \leq 2$ indicates that the model of interest is consistent with the reference model, $2 \leq \Delta \mathrm{BIC} \leq 6$ implies the positive evidence against the model, and $\triangle \mathrm{BIC} \geq 10$ suggests that such evidence becomes strong.

Let us highlight key results of our study in the following. In figure 1 and 2 , we show the $68.3 \%$ and $95.4 \%$ confidence contours for the hairy and the hairless BH models, respectively, along with the 1D marginalized likelihood for various parameter combinations. The figures, as well as the table, seem to show that our models explain the observational data as good as the $\Lambda \mathrm{CDM}$ model does. Moreover, as is seen in table 1, we find that the relative difference $2<\Delta$ AIC $<7$ and which suggests a positive evidence against our model (both hairy and hairless cases). However, if we take the smallness of AIC and BIC into an account, $\Lambda \mathrm{CDM}$ model is still favored over our model. The $\Delta \mathrm{BIC}$ values presented in table 1 indicate that, if more data is used, $\Delta$ AIC between the two models might be increasing in some extent. Thus, more data can tell us how well these models relatively fit the observational data.

In figure 3 , and using the best-fit values from table 1 , we plot the low-redshift evolution of the Hubble parameter in our models (4.2). As is seen in the figure, our models can explain the observational data [48] as good as the $\Lambda \mathrm{CDM}$ model does in the redshift $0 \leq z \leq 2.36$ interval, and the deviation from the $\Lambda \mathrm{CDM}$ model noticeable in the redshift increasing direction. We also find the redshift $z_{d a}$ of the cosmological deceleration-acceleration transition at $z_{d a}^{\Lambda \mathrm{CDM}} \simeq 0.7125, z_{d a}^{\text {Hairless }} \simeq 0.7091$, and $z_{d a}^{\text {Hairy }} \simeq 0.6954$ for each cosmological models we discuss in this study. Here, the $z_{d a}$ indicates the time that our universe transitioned from non-relativistic (baryon and cold dark) matter dominated phase to the current dark energy dominated phase; hence, $\ddot{a}=0$ at $z_{d a}$. The result in figure 3 indicates that our universe entered the phase of cosmic acceleration slightly later in the holographic models than the $\Lambda \mathrm{CDM}$ model. Thus, in order to explain the current observational data as good as the $\Lambda$ CDM model does, our two holographic models develop a faster expansion rate, a larger $H(z)$, at each redshift after the time of deceleration-acceleration transition; hence a longer history of our Universe to the CMB time.

\section{Conclusion}

We studied the background cosmological evolution from the four-dimensional boundary of a five-dimensional Anti-de Sitter $\left(A d S_{5}\right)$ black holes in this work. The four-dimensional conformal boundary takes the FRW geometry with a scale factor, $a(\tau)$. To see the FRW 


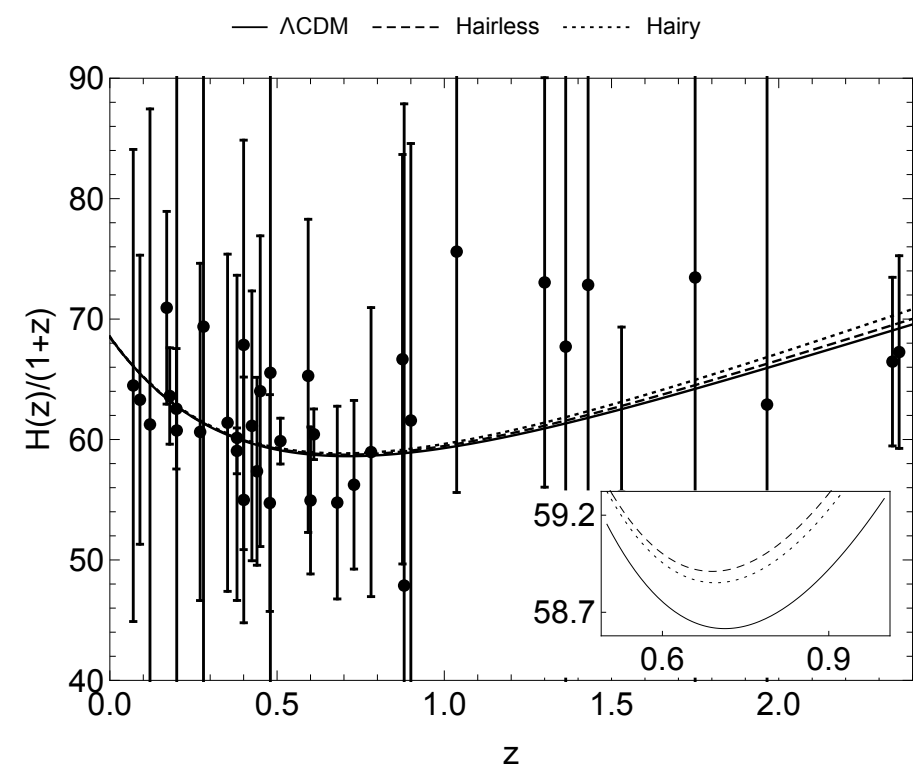

Figure 3. The redshift evolution of (4.2) in light of the observational data [48], where the numerical inputs for each model are from table 1 . The $\Lambda$ CDM model with $H(z)=H_{0} \sqrt{1-\Omega_{m}+\Omega_{m}(1+z)^{3}}$ is reflected in the solid black line.

spacetime on the boundary, we employed so-called EF coordinates and used the scaleinvariant property of the bulk geometry.

Modified Friedmann equations, which is our main result of this work, are derived on the FRW boundary of an $A d S_{5}$ BH through the AdS/CFT correspondence and its background evolution has further investigated. Since the late-time accelerating universe is our main concern here, we treated the extra contributions coming from the bulk side as dark energy and performed MCMC analysis using observational data. Compared to the $\Lambda$ CDM, our models contain additional free parameters that are associated to the charge $Q$ and mass $M$ of the BH. Thus, to make a fair comparison, we have used AIC and BIC in our analysis. Albeit the forms of equations we derived look far different from that in the standard model of cosmology, the cosmological evolution of the universe for our model found to be similar to that of the $\Lambda \mathrm{CDM}$ model. The connection with braneworld models can be found in ref. [64]

The key results of our numerical work are presented in table 1 and figure $1-3$. The figures, as well as the table, have shown that our models explain the observational data as good as the $\Lambda$ CDM model does for the current data. However, if we take the smallness of $\triangle \mathrm{AIC}$ and $\triangle \mathrm{BIC}$ into an account, $\Lambda \mathrm{CDM}$ model is still favored over our model. Moreover, the $\triangle \mathrm{BIC}$ values presented in table 1 indicate that, if more data is used, $\Delta \mathrm{AIC}$ between the two models might be increasing in some extent. Thus, more data can tell us how well these models relatively fit the observational data.

\section{Acknowledgments}

We thank Yoobin Jeong for his contribution at the initiation of the project and Yun-Long Zhang for his helpful discussions and valuable comments on an earlier version of the 
manuscript. SK was supported by Higher Education Improvement Project (HEIP) funded by the Cambodian Government (IDA Credit No. 6221-KH) and Swedish International Development Cooperation Agency (SIDA) through Sweden and Royal University of Phnom Penh (RUPP)'s Pilot Research Cooperation Programme (Sida Contribution No. 11599). BHL was supported by Basic Science Research Program through the National Research Foundation of Korea(NRF) 2020R1A6A1A03047877 and also by 2020R1F1A1075472. GT was supported by Ministry of Science and Technology (MoST) grant No. 109-2112-M-002-019.

Open Access. This article is distributed under the terms of the Creative Commons Attribution License (CC-BY 4.0), which permits any use, distribution and reproduction in any medium, provided the original author(s) and source are credited.

\section{References}

[1] I. Zlatev, L.-M. Wang and P.J. Steinhardt, Quintessence, cosmic coincidence, and the cosmological constant, Phys. Rev. Lett. 82 (1999) 896 [astro-ph/9807002] [INSPIRE].

[2] P.J. Steinhardt, L.-M. Wang and I. Zlatev, Cosmological tracking solutions, Phys. Rev. D 59 (1999) 123504 [astro-ph/9812313] [INSPIRE].

[3] X. Zhang, Coupled quintessence in a power-law case and the cosmic coincidence problem, Mod. Phys. Lett. A 20 (2005) 2575 [astro-ph/0503072] [INSPIRE].

[4] X. Zhang, Statefinder diagnostic for coupled quintessence, Phys. Lett. B 611 (2005) 1 [astro-ph/0503075] [INSPIRE].

[5] M. Chevallier and D. Polarski, Accelerating universes with scaling dark matter, Int. J. Mod. Phys. D 10 (2001) 213 [gr-qc/0009008] [InSPIRE].

[6] E.V. Linder, Exploring the expansion history of the universe, Phys. Rev. Lett. 90 (2003) 091301 [astro-ph/0208512] [INSPIRE].

[7] A.G. Cohen, D.B. Kaplan and A.E. Nelson, Effective field theory, black holes, and the cosmological constant, Phys. Rev. Lett. 82 (1999) 4971 [hep-th/9803132] [INSPIRE].

[8] M. Li, A model of holographic dark energy, Phys. Lett. B 603 (2004) 1 [hep-th/0403127] [INSPIRE].

[9] X. Zhang and F.-Q. Wu, Constraints on holographic dark energy from Type Ia supernova observations, Phys. Rev. D 72 (2005) 043524 [astro-ph/0506310] [INSPIRE].

[10] S. Nojiri and S.D. Odintsov, Unifying phantom inflation with late-time acceleration: Scalar phantom-non-phantom transition model and generalized holographic dark energy, Gen. Rel. Grav. 38 (2006) 1285 [hep-th/0506212] [INSPIRE].

[11] H. Wei and R.-G. Cai, A New Model of Agegraphic Dark Energy, Phys. Lett. B 660 (2008) 113 [arXiv: 0708.0884] [INSPIRE].

[12] C. Gao, F. Wu, X. Chen and Y.-G. Shen, A Holographic Dark Energy Model from Ricci Scalar Curvature, Phys. Rev. D 79 (2009) 043511 [arXiv:0712.1394] [INSPIRE].

[13] C. Feng, B. Wang, Y. Gong and R.-K. Su, Testing the viability of the interacting holographic dark energy model by using combined observational constraints, JCAP 09 (2007) 005 [arXiv:0706.4033] [INSPIRE]. 
[14] A. Sheykhi and M.R. Setare, Interacting new agegraphic viscous dark energy with varying $G$, Int. J. Theor. Phys. 49 (2010) 2777 [arXiv:1003.1109] [INSPIRE].

[15] R. D'Agostino, Holographic dark energy from nonadditive entropy: cosmological perturbations and observational constraints, Phys. Rev. D 99 (2019) 103524 [arXiv:1903.03836] [INSPIRE].

[16] M. Li, X.-D. Li, S. Wang and X. Zhang, Holographic dark energy models: A comparison from the latest observational data, JCAP 06 (2009) 036 [arXiv:0904.0928] [INSPIRE].

[17] R.-G. Cai, S. Khimphun, B.-H. Lee, S. Sun, G. Tumurtushaa and Y.-L. Zhang, Emergent Dark Universe and the Swampland Criteria, Phys. Dark Univ. 26 (2019) 100387

[arXiv: 1812.11105] [INSPIRE].

[18] G.R. Dvali, G. Gabadadze and M. Porrati, 4-D gravity on a brane in 5-D Minkowski space, Phys. Lett. B 485 (2000) 208 [hep-th/0005016] [INSPIRE].

[19] A.Y. Kamenshchik, U. Moschella and V. Pasquier, An alternative to quintessence, Phys. Lett. B 511 (2001) 265 [gr-qc/0103004] [INSPIRE].

[20] M.C. Bento, O. Bertolami and A.A. Sen, Generalized Chaplygin gas, accelerated expansion and dark energy matter unification, Phys. Rev. D 66 (2002) 043507 [gr-qc/0202064] [INSPIRE].

[21] K. Bamba, S. Capozziello, S. Nojiri and S.D. Odintsov, Dark energy cosmology: the equivalent description via different theoretical models and cosmography tests, Astrophys. Space Sci. $\mathbf{3 4 2}$ (2012) 155 [arXiv: 1205.3421] [INSPIRE].

[22] Y.-Y. Xu and X. Zhang, Comparison of dark energy models after Planck 2015, Eur. Phys. J. C 76 (2016) 588 [arXiv: 1607.06262] [INSPIRE].

[23] S. Wen, S. Wang and X. Luo, Comparing dark energy models with current observational data, JCAP 07 (2018) 011 [arXiv: 1708.03143] [INSPIRE].

[24] W. Yang, S. Pan, S. Vagnozzi, E. Di Valentino, D.F. Mota and S. Capozziello, Dawn of the dark: unified dark sectors and the EDGES Cosmic Dawn 21-cm signal, JCAP 11 (2019) 044 [arXiv: 1907.05344] [INSPIRE].

[25] B. Li, D.F. Mota and D.J. Shaw, Microscopic and Macroscopic Behaviors of Palatini Modified Gravity Theories, Phys. Rev. D 78 (2008) 064018 [arXiv:0805.3428] [InSPIRE].

[26] J. Valiviita, E. Majerotto and R. Maartens, Instability in interacting dark energy and dark matter fluids, JCAP 07 (2008) 020 [arXiv:0804.0232] [INSPIRE].

[27] M.B. Gavela, D. Hernandez, L. Lopez Honorez, O. Mena and S. Rigolin, Dark coupling, JCAP 07 (2009) 034 [Erratum ibid. 05 (2010) E01] [arXiv:0901.1611] [InSPIRE].

[28] A. De Felice, D.F. Mota and S. Tsujikawa, Matter instabilities in general Gauss-Bonnet gravity, Phys. Rev. D 81 (2010) 023532 [arXiv:0911.1811] [INSPIRE].

[29] T. Karwal and M. Kamionkowski, Dark energy at early times, the Hubble parameter, and the string axiverse, Phys. Rev. D 94 (2016) 103523 [arXiv: 1608.01309] [INSPIRE].

[30] J. Solà, A. Gómez-Valent and J. de Cruz Pérez, The $H_{0}$ tension in light of vacuum dynamics in the Universe, Phys. Lett. B 774 (2017) 317 [arXiv:1705.06723] [InSPIRE].

[31] E. Di Valentino, E.V. Linder and A. Melchiorri, Vacuum phase transition solves the $H_{0}$ tension, Phys. Rev. D 97 (2018) 043528 [arXiv:1710.02153] [InSPIRE].

[32] E. Di Valentino, A. Melchiorri, O. Mena and S. Vagnozzi, Nonminimal dark sector physics and cosmological tensions, Phys. Rev. D 101 (2020) 063502 [arXiv:1910.09853] [INSPIRE]. 
[33] I. Savonije and E.P. Verlinde, CFT and entropy on the brane, Phys. Lett. B 507 (2001) 305 [hep-th/0102042] [INSPIRE].

[34] A.J.M. Medved, CFT on the brane with a Reissner-Nordstrom-de Sitter twist, hep-th/0111182 [INSPIRE].

[35] S. Nojiri and S.D. Odintsov, AdS/CFT and quantum corrected brane entropy, Class. Quant. Grav. 18 (2001) 5227 [hep-th/0103078] [INSPIRE].

[36] S. Nojiri, S.D. Odintsov and S. Ogushi, Friedmann-Robertson-Walker brane cosmological equations from the five-dimensional bulk (A)dS black hole, Int. J. Mod. Phys. A 17 (2002) 4809 [hep-th/0205187] [INSPIRE].

[37] P.S. Apostolopoulos, G. Siopsis and N. Tetradis, Cosmology from an AdS Schwarzschild black hole via holography, Phys. Rev. Lett. 102 (2009) 151301 [arXiv:0809.3505] [INSPIRE].

[38] S. Banerjee, S. Bhowmick, A. Sahay and G. Siopsis, Generalized Holographic Cosmology, Class. Quant. Grav. 30 (2013) 075022 [arXiv: 1207.2983] [INSPIRE].

[39] G. Camilo, Expanding plasmas from Anti de Sitter black holes, Eur. Phys. J. C 76 (2016) 682 [arXiv: 1609.07116] [INSPIRE].

[40] G.W. Gibbons and S.W. Hawking, Action Integrals and Partition Functions in Quantum Gravity, Phys. Rev. D 15 (1977) 2752 [inSPIRE].

[41] G. Compere and D. Marolf, Setting the boundary free in AdS/CFT, Class. Quant. Grav. 25 (2008) 195014 [arXiv:0805.1902] [INSPIRE].

[42] I.R. Klebanov and E. Witten, AdS/CFT correspondence and symmetry breaking, Nucl. Phys. $B \mathbf{5 5 6}$ (1999) 89 [hep-th/9905104] [INSPIRE].

[43] S. de Haro, S.N. Solodukhin and K. Skenderis, Holographic reconstruction of space-time and renormalization in the AdS/CFT correspondence, Commun. Math. Phys. 217 (2001) 595 [hep-th/0002230] [INSPIRE].

[44] M. Bianchi, D.Z. Freedman and K. Skenderis, Holographic renormalization, Nucl. Phys. B 631 (2002) 159 [hep-th/0112119] [INSPIRE].

[45] B. Sahoo and H.-U. Yee, Electrified plasma in AdS/CFT correspondence, JHEP 11 (2010) 095 [arXiv: 1004.3541] [INSPIRE].

[46] D.M. Scolnic et al., The Complete Light-curve Sample of Spectroscopically Confirmed SNe Ia from Pan-STARRS1 and Cosmological Constraints from the Combined Pantheon Sample, Astrophys. J. 859 (2018) 101 [arXiv:1710.00845] [INSPIRE].

[47] U. Alam and J. Lasue, An Exploration of Heterogeneity in Supernova Type Ia Samples, JCAP 06 (2017) 034 [arXiv:1701.02065] [INSPIRE].

[48] M. Moresco et al., A 6\% measurement of the Hubble parameter at $z \sim 0.45$ : direct evidence of the epoch of cosmic re-acceleration, JCAP 05 (2016) 014 [arXiv: 1601.01701] [INSPIRE].

[49] R.-Y. Guo and X. Zhang, Constraining dark energy with Hubble parameter measurements: an analysis including future redshift-drift observations, Eur. Phys. J. C 76 (2016) 163 [arXiv: 1512.07703] [INSPIRE].

[50] S.S. Gubser and F.D. Rocha, Peculiar properties of a charged dilatonic black hole in AdS $S_{5}$, Phys. Rev. D 81 (2010) 046001 [arXiv:0911.2898] [INSPIRE]. 
[51] SNLS collaboration, Supernova Constraints and Systematic Uncertainties from the First 3 Years of the Supernova Legacy Survey, Astrophys. J. Suppl. 192 (2011) 1 [arXiv:1104.1443] [INSPIRE].

[52] S. Basilakos and S. Nesseris, Testing Einstein's gravity and dark energy with growth of matter perturbations: Indications for new physics?, Phys. Rev. D 94 (2016) 123525 [arXiv: 1610.00160] [INSPIRE].

[53] M. Goliath, R. Amanullah, P. Astier, A. Goobar and R. Pain, Supernovae and the nature of the dark energy, Astron. Astrophys. 380 (2001) 6 [astro-ph/0104009] [INSPIRE].

[54] R. Lazkoz, S. Nesseris and L. Perivolaropoulos, Comparison of Standard Ruler and Standard Candle constraints on Dark Energy Models, JCAP 07 (2008) 012 [arXiv:0712.1232] [INSPIRE].

[55] J.C.B. Sanchez, S. Nesseris and L. Perivolaropoulos, Comparison of Recent SnIa datasets, JCAP 11 (2009) 029 [arXiv:0908.2636] [INSPIRE].

[56] S. Nesseris, A. De Felice and S. Tsujikawa, Observational constraints on Galileon cosmology, Phys. Rev. D 82 (2010) 124054 [arXiv:1010.0407] [InSPIRE].

[57] A. De Felice, S. Nesseris and S. Tsujikawa, Observational constraints on dark energy with a fast varying equation of state, JCAP 05 (2012) 029 [arXiv:1203.6760] [INSPIRE].

[58] B.S. Kim, Holographic Renormalization of Einstein-Maxwell-Dilaton Theories, JHEP 11 (2016) 044 [arXiv: 1608.06252] [INSPIRE].

[59] Y. Jeong, S. Khimphun, B.-H. Lee and G. Tumurtushaa, Dark Energy Constraints from a five-dimensional AdS Black Hole via AdS/CFT, EPJ Web Conf. 206 (2019) 09007 [INSPIRE].

[60] E. Gaztanaga, A. Cabre and L. Hui, Clustering of Luminous Red Galaxies IV: Baryon Acoustic Peak in the Line-of-Sight Direction and a Direct Measurement of H(z), Mon. Not. Roy. Astron. Soc. 399 (2009) 1663 [arXiv:0807.3551] [InSPIRE].

[61] R. Jimenez and A. Loeb, Constraining cosmological parameters based on relative galaxy ages, Astrophys. J. 573 (2002) 37 [astro-ph/0106145] [INSPIRE].

[62] H. Akaike, A new look at the statistical model identification, IEEE Trans. Automat. Contr. 19 (1974) 716.

[63] G. Schwarz, Estimating the Dimension of a Model, Annals Statist. 6 (1978) 461.

[64] D. Huang, B.-H. Lee, G. Tumurtushaa, L. Yin and Y.-L. Zhang, Observational Constraints on the Cosmology with Holographic Dark Fluid, Phys. Dark Univ. 32 (2021) 100842 [arXiv:2101.02978] [INSPIRE]. 